\title{
The Gap in Prescribing Stroke Prevention Therapies in Postoperative Atrial Fibrillation After Isolated Coronary Artery Bypass Grafting Surgery
}

\author{
Mohammed Shurrab, MD, MSc, ${ }^{1,2,3,4}$ Dennis T. Ko, MD, MSc, ${ }^{4,5,6}$ Rony Atoui, MD, MSc, ${ }^{2,3,7}$ Kari Kostiw, NP, \\ Mark Henderson, $\mathrm{MD}^{1,3}$ \\ ${ }^{1}$ Cardiology Department, Health Sciences North, Sudbury, Ontario, Canada; ${ }^{2}$ Health Sciences North Research Institute, Sudbury, \\ Ontario, Canada; ${ }^{3}$ Northern Ontario School of Medicine, Laurentian University, Sudbury, Ontario, Canada; ${ }^{4}$ Institute of Health Policy, \\ Management and Evaluation, University of Toronto, Toronto, Ontario, Canada; ${ }^{5}$ Division of Cardiology, Schulich Heart Centre, \\ Sunnybrook Health Sciences Centre, University of Toronto, Toronto, Ontario, Canada; ${ }^{6}$ Institute for Clinical Evaluative Sciences, Toronto, \\ Ontario, Canada; ${ }^{7}$ Division of Cardiac Surgery, Health Sciences North, Sudbury, Ontario, Canada
}

\section{ABSTRACT}

Background: Postoperative atrial fibrillation (POAF) commonly occurs after coronary artery bypass graft (CABG) surgery. Patients with POAF are at higher risk of stroke and mortality. The use of oral anticoagulation (OAC) in POAF remains inconsistent, and the care gap is unknown. Objective: We sought to evaluate rate of adherence and factors associated with OAC use in POAF patients after isolated CABG.

Methods: In this study, all patients who had an isolated CABG between April 1, 2019 and March 30, 2020, at Health Sciences North, Sudbury retrospectively were evaluated. We identified all patients who developed POAF and captured the use of OAC. Multivariable logistic regression models were used to identify predictors of OAC prescription.

Results: In total, 339 CABG patients between April 1, 2019 and March 30, 2020, were identified; 86 patients developed POAF. No patients had major perioperative bleeding. All POAF patients had an indication for OAC therapies based on CHADS 65 score. However, only 17 (19.8\%) patients with POAF had OAC prescription at the time of hospital discharge. Baseline characteristics were similar between those with or without OAC. In multivariable analysis, the use of dual antiplatelet therapy was associated with a decreased use of OAC therapy in POAF (OR 0.037 (95\% CI 0.005, 0.29), $P=.002)$. Moreover, in comparison to surgeon $\mathrm{A}$, surgeon $\mathrm{B}$ was associated with a decreased use of OAC therapy, whereas surgeon $\mathrm{C}$ was associated with an increased use of OAC therapy in POAF (OR 0.15 (95\% CI 0.03, 0.83), $P=.03$ and OR 6.49 (95\% CI 1.21, 34.82), $P=.03$, respectively). None of the CHADS 65 elements, including age, hypertension, stroke, diabetes mellitus or congestive heart failure, correlated with the use of OAC.

Received March 31, 2021; received in revised form April 19, 2021; accepted May 2, 2021.

Correspondence: Mohammed Shurrab, MD, MSc, Cardiology Department, Health Sciences North, Health Sciences North Research Institute, Northern Ontario School of Medicine Laurentian University, Sudbury, Ontario, Canada (e-mail: shurrabm@botmail.com).
Key findings: Eighty percent of patients who developed POAF after isolated CABG failed to receive stroke prevention therapies. The use of dual antiplatelet therapy and individual surgeons' preference were associated with the use of OAC therapy among POAF.

Conclusions: This data set suggests that approximately only 1 in 5 patients with POAF after isolated CABG got prescribed OAC.

\section{INTRODUCTION}

Coronary artery bypass grafting (CABG) surgery is the most common type of cardiac surgery performed worldwide [Rocha 2017]. Postoperative atrial fibrillation (POAF) after CABG is common, occurring between 20-40\% [Benedetto 2020; Philip 2014]. POAF typically occurs on postoperative day 2, with $70 \%$ of cases occurring within the first four postoperative days [Benedetto 2020; Philip 2014]. Multiple studies have shown that POAF patients are at higher risk of stroke and mortality. A recent study found the incidence of cerebrovascular accident (CVA) is $6.3 \%$ at 10 years follow up in POAF patients after an isolated CABG [Benedetto 2020]. This is in line with the results of a meta-analysis of new-onset $\mathrm{AF}$ after $\mathrm{CABG}$, where the presence of POAF was associated with increased long-term risk of stroke compared with patients without POAF [Megens 2017].

Although recent evidence highlights the stroke risk related to POAF after isolated CABG, the practice of prescribing oral anticoagulation $(\mathrm{OAC})$ therapies remains heterogenous and inconsistent. This is multifactorial and related to the notion that POAF usually is transient and hence does not necessarily require OAC therapy. In fact, despite increasing evidence of late recurrence of $\mathrm{AF}$ in these patients, most physicians delay starting anticoagulation to avoid complications related to bleeding [Kotecha 2020; Woldendorp 2020]. Moreover, the current guidelines recommend anticoagulation for patients with a prolonged duration of POAF ( $>24-48$ hours) for at least four weeks [January 2014; January 2019; Sousa-Uva 2018]. Nevertheless, the recent literature has shown the association of stroke to POAF with much shorter $\mathrm{AF}$ duration, where some studies included AF of at least 30 seconds duration [Benedetto 2020]. 


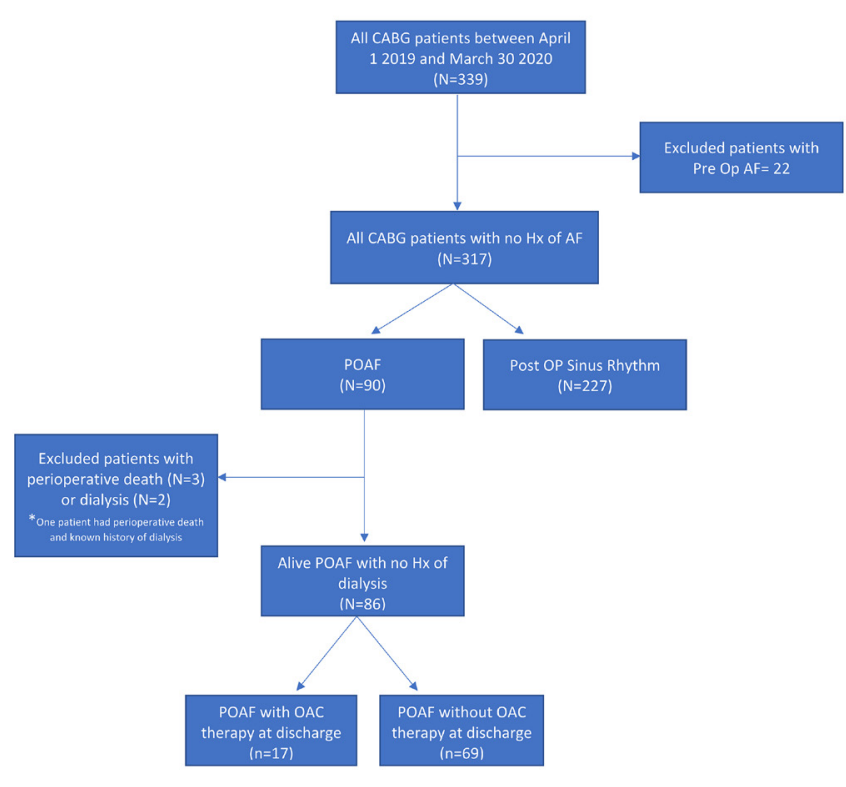

Cohort creation

Multiple recommendations, regarding the perioperative management of AF, recently have been released from several cardiovascular societies. Despite this, there remains a heterogeneity in the prescription of OAC postoperatively as recently highlighted by Schaffer et al. [Schaffer 2019] in a prospective cohort study analyzing more than 70000 patients, including patients who had cardiac surgery. They noted a low level of OAC prescriptions in their cohort, even among high risk. This finding also was highlighted in another recent study led by the Society of Cardiovascular Anesthesiologists and European Association of Cardiothoracic Anesthetists [O'Brien 2019]. In their study, they analyzed the results of an evidence-based set of survey questions to better highlight the differences in the management of perioperative and postoperative AF management in North America and Europe. The survey suggested that despite awareness of current guideline recommendations, including class I recommendations, most responding surgeons selectively follow these recommendations highlighting again the spectrum of adherence to the published guidelines. Fortunately, initiation of anticoagulation has been increasing over time with the use of direct oral anticoagulants (DOACs) as highlighted by a recent Society of Thoracic Surgeons (STS) database study analyzing $>30000$ cardiac surgery patients [Beller 2020].

We sought to evaluate the rate of adherence and the factors associated with OAC use in POAF patients after isolated CABG surgery in a tertiary hospital in Northern Ontario.

\section{METHODS}

Study design and data sources: In this study, all consecutive patients who had an isolated CABG between April 1, 2019 and March 30, 2020, at Health Sciences North, Sudbury retrospectively were evaluated as part of a quality improvement
Table 1S. Baseline Characteristics in Patients With and Without Postoperative Atrial Fibrillation after Coronary Artery Bypass Graft Surgery

\begin{tabular}{lccc}
\hline & $\begin{array}{c}\text { Postoperative } \\
\text { Atrial Fibrillation } \\
\text { Predictor }\end{array}$ & $\begin{array}{c}\text { Sinus Rhythm } \\
(\mathrm{N}=90)\end{array}$ & \\
\hline Age (mean, IQR) & $71(66-77)$ & $65(58-71)$ & $<.0001 \S$ \\
Sex (\# of female, $\mathrm{n}, \%)$ & $15(16.7)$ & $61(26.9)$ & $.059 *$ \\
CHF (n, \%) & $35(38.9)$ & $61(26.9)$ & $.042^{*}$ \\
HTN (n, \%) & $84(93.3)$ & $216(95.2)$ & $.58^{*}$ \\
DM (n, \%) & $49(54.4)$ & $124(54.6)$ & $.99 *$ \\
History of stroke (n, \%) & $10(11.1)$ & $18(7.9)$ & $.38 *$ \\
LVEF (median, IQR) & $55(49-62)$ & $58(52-63)$ & $.13 \S$ \\
OAC Therapy (n, \%) & $17(18.9)$ & $2(0.9)$ & $<.0001 \#$ \\
\hline
\end{tabular}

*Chi-square test, \#Fisher's exact test, §Wilcoxon Rank Sum, IQR, interquartile range;

$\mathrm{CHF}$, congestive heart failure; DM, diabetes mellitus; HTN, hypertension; OAC, oral anticoagulation; LVEF, left ventricular ejection fraction

project. Data extraction was performed from electronic medical records (EMR). Also, a comprehensive chart review was performed by two independent reviewers.

Data collection and study population: We included all patients who had an isolated CABG between April 1, 2019 and March 30, 2020. We collected all data available related to baseline characteristics along with OAC therapies prescriptions at discharge. In line with the aims of this study, we excluded all patients with preoperative AF. We excluded any patient with a known history of AF or who had preoperative documentation of AF.

We identified all patients who developed POAF. This was performed by reviewing EMR and a manual chart review of all records and available ECGs and telemetry strips. This was confirmed by two independent reviewers. POAF was defined as the occurrence of any episode of AF or flutter after the index procedure through the time of discharge that lasted at least six minutes. This definition has been used in major $\mathrm{AF}$ clinical trials [Healey 2012]. We also stratified AF episodes with the 24 hours duration as a cut off. This was performed using the following tools: 1) ECG lab report results confirming diagnosis of AF, 2) cardiac telemetry tracing in the chart review, 3) consult note from a specialist confirming diagnosis, 4) discharge note/report with $\mathrm{AF}$ as a listed diagnosis, 5) nurse/allied health care note with $\mathrm{AF}$ as a listed diagnosis in the chart, and 6) EMR list of diagnoses includes AF. Any of those tools were used to identify patients with $\mathrm{AF}$, and then we reviewed telemetry to capture AF durations and confirm the diagnosis.

Among patients with POAF, we excluded patients with perioperative death or those on dialysis, which could preclude the prescription of an OAC. Postoperative bleeding was assessed in all patients with POAF. This was defined as 
Table 1. Baseline Characteristics in Postoperative Atrial Fibrillation Patients With and Without OAC Therapy at Discharge

\begin{tabular}{lccc}
\hline Predictor & $\begin{array}{c}\text { With OAC } \\
\text { Therapy (N=17) }\end{array}$ & $\begin{array}{c}\text { Without OAC } \\
\text { Therapy (N=69) }\end{array}$ & $P$ \\
\hline Age (median, IQR) & $69(67-75)$ & $72(67-77)$ & $.46 \S$ \\
Sex (\# of female, $\mathrm{n}, \%)$ & $1(5.9)$ & $13(18.8)$ & $.28 \#$ \\
CHF (n, \%) & $8(47.1)$ & $24(34.8)$ & $.41 *$ \\
HTN (n, \%) & $16(94.1)$ & $65(94.2)$ & $>.99 *$ \\
DM (n, \%) & $10(58.8)$ & $37(53.6)$ & $.79 *$ \\
History of stroke (n, \%) & $2(11.8)$ & $8(11.6)$ & $>.99 \#$ \\
LVEF (median, IQR) & $52(47-60)$ & $56(50-62)$ & $.23 \S$ \\
Surgeon A & $4(23.5)$ & $26(37.7)$ & $.39 \#$ \\
Surgeon B & $5(29.4)$ & $23(33.3)$ & $.22 \#$ \\
Surgeon C & $8(47.1)$ & $19(27.5)$ & $.15 *$ \\
AF duration >24 & $16(94.1)$ & $51(73.9)$ & $.1 *$ \\
Cardioversion & $0(0 \%)$ & $1(1.5)$ & $>.99 \#$ \\
Single Antiplatelet & $17(100)$ & $67(97)$ & $>.99 *$ \\
Dual Antiplatelet & $3(17.7)$ & $42(60.9)$ & $.002 \#$ \\
\hline
\end{tabular}

*Chi-square test, §Wilcoxon Rank Sum, \# Fisher's exact test; IQR, interquartile range;

CHF, congestive heart failure; DM, diabetes mellitus; HTN, hypertension;

OAC, oral anticoagulation; LVEF, left ventricular ejection fraction

major bleeding that required intervention. Cohort creation is detailed in Figure.

This research initiative was reviewed and approved as a quality improvement project and approved by the research office at Health Sciences North Research Institute. The data were gathered by health care providers within the circle of care, de-identified and only presented in aggregate. Informed consent was therefore not required.

Outcomes and covariates: We examined the magnitude and predictors of the gap between indicated and prescribed OAC therapies in POAF patients after isolated CABG surgery.

Several covariates were examined in this study related to patients' demographics, comorbidities and relevant medications. Patient demographic characteristics included age and sex. Elements of CHADS 65 and possible reasons for prescribing $\mathrm{OAC}$ therapies in $\mathrm{AF}$ were examined and included the following: congestive heart failure (CHF), diabetes mellitus (DM), hypertension (HTN), and history of stroke. We captured the left ventricular ejection fraction (LVEF) as reported by the preoperative echocardiograms. We looked at surgeons' individual use of OAC therapy. We have used surgeon $\mathrm{A}$ as a reference for comparison given that this surgeon has the highest case volume. Finally, we included the use of single and dual antiplatelet therapy. The decision on the variables was based on discussion among the coauthors and clinical judgement.
Statistical analysis: Baseline characteristics were compared between those with or without OAC therapy. The normality of continuous variables was assessed by visually inspecting histograms and quantile-quantile (q-q) plots, and performing the Shapiro-Wilk test of normality. Continuous, normally distributed variables were compared using Student's T-test. For continuous non-normally distributed variables, the Wilcoxon Rank Sum test was used. Associations between categorical variables were assessed with the chi-square test or Fisher's exact test.

To identify factors associated with the use of OAC therapies, a multivariable logistic regression was constructed. Patient-related independent variables were selected $a$ priori for this model, based on discussion among coauthors and clinical judgement. Variables were assessed for collinearity, and those with a variance inflation factor $>4$ were considered to be collinear. Selection for exclusion or inclusion of collinear variables was performed on the basis of clinical judgment, study design, and objectives. We have not identified any interactions within the included variables. A $P$-value of $<0.05$ was considered to be statistically significant.

Model assumptions were verified. Model fit was assessed using the Hosmer-Lemeshow test for fit. Model overspecification was mitigated by limiting the maximum number of independent variables to the clinically important ones for our hypothesis. Potential influential outliers were verified by inspection of several casewise diagnostics plots. Analyses were conducted using SAS software.

\section{RESULTS}

Baseline characteristics - CABG cohort: In total, 339 consecutive patients undergoing isolated CABG between April 1, 2019 and March 30, 2020, at our hospital were identified. POAF was confirmed in $90(28.4 \%)$ patients. Patients with POAF were older (median 71 years (IQR 66-77) versus 65 years (IQR 58-71), $P<.0001)$, similar female rate $(15(16.7 \%)$ versus $61(26.9 \%), P=.059)$ with a higher incidence of $\mathrm{CHF}$ (35 (38.9\%) versus $61(26.9 \%), P=.042)$ but similar rate of HTN, DM, or history of stroke. There was no statistically significant difference in the preoperative LVEF among both groups (median 55\% (49-62) versus 58\% (52-63), $P=.13$ ) (Table 1S).

Baseline characteristics - POAF cohort: Among the POAF patients, we excluded patients with perioperative death $(\mathrm{N}=3)$. Also, we excluded patients on dialysis $(\mathrm{N}=2)$ that could preclude the use of OAC therapy. One patient had perioperative death and known history of dialysis. None of the patients had major bleeding that required intervention. Only one patient had cardioversion among patients with POAF. Antiplatelet therapy commonly was used among POAF with 83 patients on ASA, 46 patients on clopidogrel, and one on ticagrelor. All POAF patients had indication for OAC therapy for stroke prevention based on CHADS 65 score. Among the alive POAF with no history of dialysis $(\mathrm{N}=86)$, patients with OAC therapy had similar age (median 69 years $(67-75)$ versus 72 years $(67-77), P=.46)$ and similar female rate $(1(5.9 \%)$ 
Table 2. Association between prescribing OAC therapy at discharge and clinical predictors in postoperative atrial fibrillation patients after coronary artery bypass graft surgery

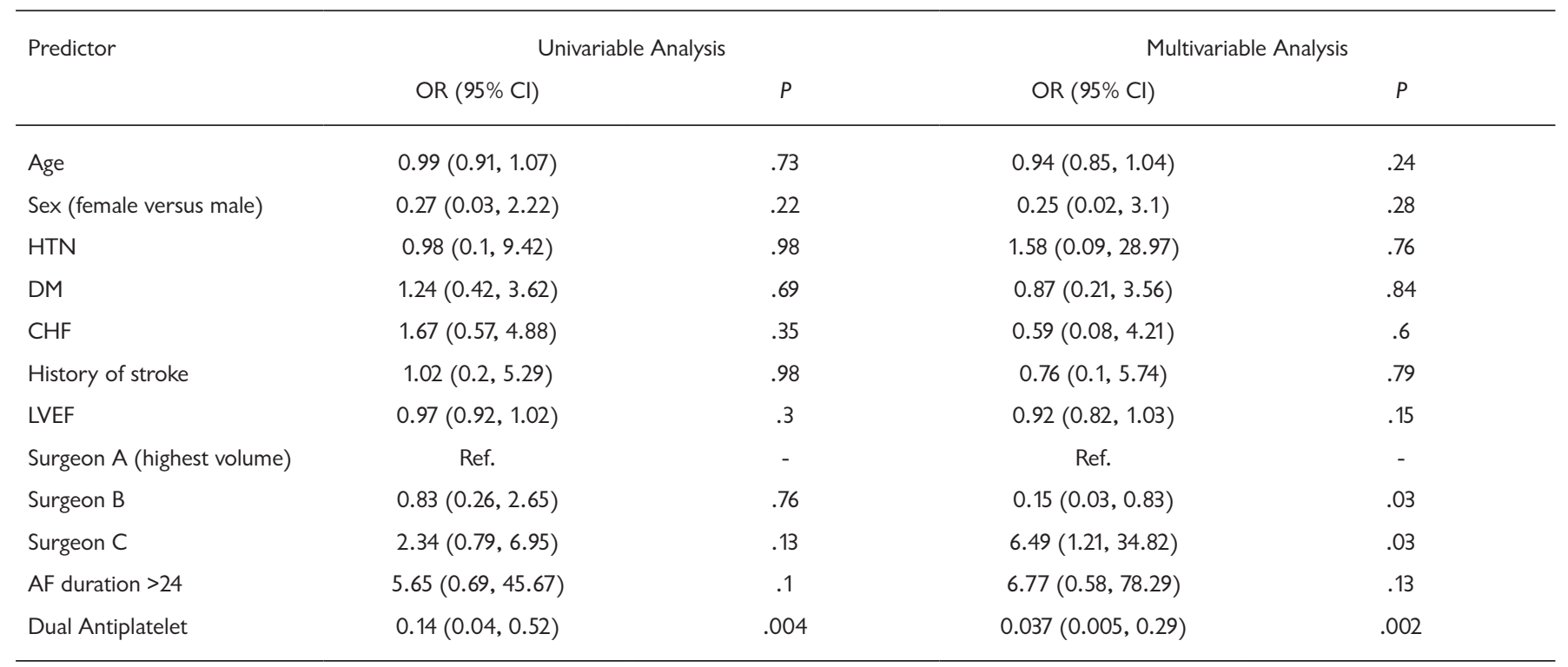

OR, odds ratio; CHF, congestive heart failure; DM, diabetes mellitus; HTN, hypertension; OAC, oral anticoagulation; LVEF, left ventricular ejection fraction

versus $13(18.8 \%), P=.28)$. POAF patient with OAC therapy had less dual antiplatelet use $(3(17.7 \%)$ versus $42(60.9 \%)$, $P=.002)$. There were no significant differences in baseline characteristics among POAF patients with or without OAC therapies, including history of HTN, DM, CHF, or history of stroke. There was similar LVEF among both groups (median $52 \%(47-60)$ versus 56\% (50-62), $P=.23$ ) (Table 1 ).

Rate and predictors of care gap: Among the POAF patients with an isolated CABG $(\mathrm{N}=86)$, only $17(19.8 \%)$ patients had OAC prescription at the time of hospital discharge, with 13 on apixaban, two on rivaroxaban, and two on warfarin. There were 24 patients in $\mathrm{AF}$ at the time of discharge, out of which nine patients were prescribed anticoagulation therapy. We identified three patients among POAF patients, who developed stroke prior to discharge. Two patients were on POD \#2 and one patient on POD \#3, and none were on anticoagulation therapy. In multivariable analysis, the use of dual antiplatelet therapy was associated with decreased use of OAC therapy in POAF (OR 0.037 (95\% CI 0.005, 0.29), $P=.002$ ). In comparison to surgeon $\mathrm{A}$, surgeon $\mathrm{B}$ was associated with a decreased use of OAC therapy, but surgeon $\mathrm{C}$ was associated with an increased use of OAC therapy in POAF (OR 0.15 (95\% CI 0.03, 0.83), $P=.03$ and OR 6.49 (95\% CI 1.21, $34.82), P=.03$, respectively). Age and sex were not associated with the use of OAC therapy (OR 0.98 (95\% CI 0.91, 1.1), $P=.71$ and OR 0.29 (95\% CI $0.035,2.47), P=.26$, respectively). AF duration (cut off 24 hours) was not associated with the use of OAC therapy (OR 6.77(95\% CI 0.58, 78.29), $P=.13)$. History of stroke was not associated with the use of OAC therapy (OR 1.1 (95\% CI $0.2,6.16), P=.91)$. Also, LVEF was not associated with the use of OAC therapy (OR 56 (95\% CI 50-62), $P=.23$ ). None of the potential attributable comorbidities consistently correlated with the use of OAC therapy (Table 2).

\section{DISCUSSION}

This study demonstrates the following important findings: 1) Eighty percent of patients who developed POAF after isolated CABG failed to receive stroke prevention therapies; and 2) The use of dual antiplatelet therapy and individual surgeons' preference were associated with the use of OAC therapy among POAF.

POAF is the most common arrhythmia following cardiac surgery and is associated with significantly higher rates of both short and long-term morbidity and mortality, including strokes, thromboembolic complications, prolonged hospital stay, and health care resource utilization [Almassi 2015; Tulla 2015; Woldendorp 2009]. POAF has shown to be an independent predictor of stroke [Benedetto 2020; Megens 2017; Ahlsson 2009; Whitlock 2014]. Benedetto et al examined the association between POAF after an isolated CABG and longterm risk of CVA at 10-year follow up. They included patients with evidence of POAF within six weeks after discharge. They found that the cumulative incidence of CVA was $6.3 \%$ versus $3.7 \%$ in patients with $\mathrm{POAF}$ and sinus rhythm, respectively [Benedetto 2020]. In a large study, all CABG patients in Sweden between 2005 and $2015(\mathrm{~N}=38040)$ retrospectively were evaluated. The incidence of POAF was $28.5 \%$ with significantly higher adjusted risk for ischemic stroke [HR 1.19 (1.091.30)], and transient ischemic attack [HR 1.17 (1.031.33)] in comparison to patients with no POAF [Bergfeldt 2019]. Multiple studies have shown similar results 
with increased risk of stroke and mortality [Megens 2017; Ahlsson 2009; Antonelli 2004; Saxena 2012; Kosmidou 2018; Mathew 2004].

There is still a significant inconsistency in the management of anticoagulation in patients with POAF. The discrepancy in the practice of prescribing OAC therapies is multifactorial and related to the notion that POAF usually is transient and hence does not necessarily require OAC therapy along with the fear of increasing bleeding risk in the postoperative course [Kotecha 2020; Woldendorp 2020]. The current guidelines recommend anticoagulation for postoperative patients with a prolonged duration of POAF ( $>24-48$ hours) for at least four weeks [January 2014; January 2019; Sousa-Uva 2018]. This is likely to be changed in subsequent recommendations given the more recent and strong evidence of the association of POAF and stroke with much shorter AF durations [Benedetto 2020]. The initiation of OAC therapies in POAF continues to be complex and physicians need to make their own decisions, taking into account the risk of stroke and risk of perioperative bleeding with no clear guidance or recommendations.

Anticoagulation following cardiac surgery requires special consideration due to the increased risk of bleeding complications in this high-risk population. Although the use of DOACs after cardiac surgery have gained popularity, their role remains controversial in postoperative cardiac surgery patients [Woldendorp 2020]. Studies comparing DOACs to warfarin for postoperative AF following $\mathrm{CABG}$ are limited in number and quality with relatively small cohort size and short length of follow ups, as highlighted in a recent literature review led by Manuel et al. [Manuel 2020], who analyzed the incidence of complications in postoperative cardiac surgery patients when using DOACs versus warfarin. They concluded that DOACs appear to be safe after cardiac surgery, although one study showed an increased incidence of pericardial and pleural effusions leading to a higher rate of reinterventions and readmissions [Yu 2019]. Similar results were obtained by Vranckx et al. [Vranckx 2016], who showed a higher perception of bleeding requiring pericardiocentesis, when DOACs were used compared to VKA in survey study performed by European Heart Rhythm Association. These findings essentially highlight the importance and need for further randomized control trials to confirm the safety of DOACs in postcardiac surgery patients and better disseminate the practice guidelines in this field.

Our cohort, similar to the literature, has shown that the incidence of POAF after an isolated CABG is $28.4 \%$. Multiple studies have used a duration of at least 30 seconds to define POAF. We chose a longer AF duration of six minutes for POAF to better identify those patients with significant episodes of AF as many of the arrhythmias below this threshold could be confused with AF with different risk profile [Healey 2012]. Also, there is a clear correlation between non-surgical AF of six minutes (or more) duration and risk of stroke [Healey 2012]. We also stratified AF episodes with the 24 hours duration as a cut off. All POAF patients had indication for OAC therapy for stroke prevention based on CHADS 65 score, and none had major bleeding. Among the POAF patients with an isolated CABG $(\mathrm{N}=86)$, only 17
$(19.8 \%)$ patients had OAC prescription at the time of hospital discharge. We examined prescriptions at the time of discharge as patients should be stable for discharge and hence physicians should feel more comfortable in initiating OAC therapies. There were 24 patients in $\mathrm{AF}$ at the time of discharge, out of which nine patients were prescribed anticoagulation therapy. Nevertheless, the literature has supported that a temporal relationship does not exist between $\mathrm{AF}$ episodes and stroke [Daoud 2011]. We identified three patients among POAF patients, who developed stroke prior to discharge two patients on POD \#2 and one patient on POD \#3 - and none were on anticoagulation therapy. Nevertheless, there is a significant care gap as $80 \%$ of POAF patients failed to receive stroke prevention therapies. In our study, the use of dual antiplatelet therapy was associated with a decreased use of OAC therapy among POAF. Certainly, one of the significant clinical challenges with OAC after CABG is that these patients often are on dual antiplatelet therapy, especially after an acute coronary event. The balance of risk versus benefits of adding OAC on top of dual antiplatelet therapy, when there is no strong evidence to support OAC use, is questionable. Dual antiplatelet therapy is not optimal for stroke prevention in patients with AF. In the ACTIVE trial, oral anticoagulation therapy was superior to clopidogrel plus aspirin for prevention of vascular events in patients with $\mathrm{AF}$ at high risk of stroke [Investigators 2006]. Also, there was an association between individual surgeons and the use of OAC therapy in POAF. This essentially highlights the lack of guidance with regard to starting anticoagulation in patients with $\mathrm{POAF}$ with the final decision ultimately being left to the treating clinicians and their own individual preferences. This highlights that part of the variability in OAC therapy that is related to surgeons' preferences even when POAF duration is longer than 24 hours.

Our study highlights the significant care gap currently seen in practice and adequately described in the literature as the 'risk-treatment' paradox, wherein patients who benefit the most from evidence-based care receive it the least [McAlister 2011; Shurrab 2017]. The significant care gap in POAF establishes a clear need for a quality improvement intervention to improve care, especially when the risk of stroke and mortality in patients with POAF is comparable to patients with nonsurgical AF [Kotecha 2020].

\section{LIMITATIONS}

The retrospective nature and relatively small number of POAF patients in our study may limit our conclusions. The decision to give anticoagulation is a balance of risk and benefits in this high-risk cohort. There may be other unmeasured covariates that determine the decision to give or withhold anticoagulation, which are not obtained from this study. The reliance of our study on medical reports also is a potential limitation. The diagnosis of AF depends highly on extensive monitoring and patients with silent AF could be missed in our study. Finally, the lack of long-term data on outcomes, such as stroke, is another limitation of this study. 
CONCLUSION

This real-world data set from a tertiary center in Northern Ontario suggests that approximately only 1 in 5 patients with POAF after isolated CABG got prescribed OAC. The use of dual antiplatelet therapy and individual surgeons' preferences were associated with the use of OAC therapy among POAF.

\section{ACKNOWLEDGEMENT}

Sources of funding: This quality improvement project was funded by an Educational Grant from the BMS/Pfizer Alliance. Ethics statement: This research initiative was reviewed and approved as a quality improvement project and approved by the research office at Health Sciences North Research Institute. The data were gathered by health care providers within the circle of care, de-identified and only presented in aggregate. Conflict of interest: Mohammed Shurrab is supported by a Fellowship Award from the Canadian Institutes of Health Research (CIHR).

\section{REFERENCES}

Ahlsson A, Bodin L, Fengsrud E and Englund A. 2009. Patients with postoperative atrial fibrillation have a doubled cardiovascular mortality. Scand Cardiovasc J. 43:330-6.

Almassi GH, Wagner TH, Carr B, Hattler B, Collins JF, Quin JA, Ebrahimi R, Grover FL, Bishawi M, Shroyer AL, and Group VAROOBS. 2015. Postoperative atrial fibrillation impacts on costs and one-year clinical outcomes: the Veterans Affairs Randomized On/Off Bypass Trial. Ann Thorac Surg. 99:109-14.

Antonelli D, Peres D, Freedberg NA, Feldman A, and Rosenfeld T. 2004. Incidence of postdischarge symptomatic paroxysmal atrial fibrillation in patients who underwent coronary artery bypass graft: long-term followup. Pacing Clin Electrophysiol. 27:365-7.

Beller JP, Krebs ED, Hawkins RB, Mehaffey JH, Quader MA, Speir AM, Kiser AC, Joseph M, Yarboro LT, Teman NR, and Ailawadi G. 2020. Non-vitamin K oral anticoagulant use after cardiac surgery is rapidly increasing. J Thorac Cardiovasc Surg. 160:1222-1231.

Benedetto U, Gaudino MF, Dimagli A, Gerry S, Gray A, Lees B, Flather M, Taggart DP, and Investigators* ART. 2020. Postoperative Atrial Fibrillation and Long-Term Risk of Stroke After Isolated Coronary Artery Bypass Graft Surgery. Circulation. 142:1320-1329.

Bergfeldt ATAJLFSNAASBL. 2019. New onset atrial fibrillation efter cardiac surgery is associated with increased longterm morbidity and mortality: a populationbased study from the SWEDEHEARTregistry. Paper presented at: European Heart Journal; October.

Daoud EG, Glotzer TV, Wyse DG, Ezekowitz MD, Hilker C, Koehler J, Ziegler PD, and Investigators T. 2011. Temporal relationship of atrial tachyarrhythmias, cerebrovascular events, and systemic emboli based on stored device data: a subgroup analysis of TRENDS. Heart Rhythm. 8:1416-23.

Healey JS, Connolly SJ, Gold MR, Israel CW, Van Gelder IC, Capucci A, Lau CP, Fain E, Yang S, Bailleul C, Morillo CA, Carlson M, Themeles E, Kaufman ES, Hohnloser SH, and Investigators A. 2012. Subclinical atrial fibrillation and the risk of stroke. N Engl J Med. 366:120-9.
Investigators AWGotA, Connolly S, Pogue J, Hart R, Pfeffer M, Hohnloser S, Chrolavicius S, Pfeffer M, Hohnloser S, and Yusuf S. 2006. Clopidogrel plus aspirin versus oral anticoagulation for atrial fibrillation in the Atrial fibrillation Clopidogrel Trial with Irbesartan for prevention of Vascular Events (ACTIVE W): a randomised controlled trial. Lancet. 367:1903-12.

January CT, Wann LS, Alpert JS, Calkins H, Cigarroa JE, Cleveland JC, Jr., Conti JB, Ellinor PT, Ezekowitz MD, Field ME, Murray KT, Sacco RL, Stevenson WG, Tchou PJ, Tracy CM, Yancy CW, and Members AATF. 2014. 2014 AHA/ACC/HRS guideline for the management of patients with atrial fibrillation: a report of the American College of Cardiology/American Heart Association Task Force on practice guidelines and the Heart Rhythm Society. Circulation. 130:e199-267.

January CT, Wann LS, Calkins H, Chen LY, Cigarroa JE, Cleveland JC, Jr., Ellinor PT, Ezekowitz MD, Field ME, Furie KL, Heidenreich PA, Murray KT, Shea JB, Tracy CM, and Yancy CW. 2019. 2019 AHA/ACC/ HRS Focused Update of the 2014 AHA/ACC/HRS Guideline for the Management of Patients With Atrial Fibrillation: A Report of the American College of Cardiology/American Heart Association Task Force on Clinical Practice Guidelines and the Heart Rhythm Society in Collaboration With the Society of Thoracic Surgeons. Circulation. 140:e125-e151.

Kosmidou I, Chen S, Kappetein AP, Serruys PW, Gersh BJ, Puskas JD, Kandzari DE, Taggart DP, Morice MC, Buszman PE, Bochenek A, Schampaert E, Page P, Sabik JF, 3rd, McAndrew T, Redfors B, BenYehuda O, and Stone GW. 2018. New-Onset Atrial Fibrillation After PCI or CABG for Left Main Disease: The EXCEL Trial. J Am Coll Cardiol. 71:739-748.

Kotecha D and Castella M. 2020. Is it time to treat postoperative atrial fibrillation just like regular atrial fibrillation? Eur Heart J. 41:652-654a.

Manuel L, Fong LS, Ang ZH, and Grant P. 2020. Comparison of novel oral anticoagulants versus warfarin for post-operative atrial fibrillation after coronary artery bypass grafting. Ann Med Surg (Lond). 58:130-133.

Mathew JP, Fontes ML, Tudor IC, Ramsay J, Duke P, Mazer CD, Barash PG, Hsu PH, Mangano DT, Investigators of the Ischemia R, Education F and Multicenter Study of Perioperative Ischemia Research G. 2004. A multicenter risk index for atrial fibrillation after cardiac surgery. JAMA. 291:1720-9.

McAlister FA. 2011. The end of the risk-treatment paradox? A rising tide lifts all boats. J Am Coll Cardiol. 58:1766-7.

Megens MR, Churilov L, and Thijs V. 2017. New-Onset Atrial Fibrillation After Coronary Artery Bypass Graft and Long-Term Risk of Stroke: A Meta-Analysis. J Am Heart Assoc. 6.

O'Brien B, Burrage PS, Ngai JY, Prutkin JM, Huang CC, Xu X, Chae SH, Bollen BA, Piccini JP, Schwann NM, Mahajan A, Ruel M, Body SC, Sellke FW, Mathew J, and Muehlschlegel JD. 2019. Society of Cardiovascular Anesthesiologists/European Association of Cardiothoracic Anaesthetists Practice Advisory for the Management of Perioperative Atrial Fibrillation in Patients Undergoing Cardiac Surgery. J Cardiothorac Vasc Anesth. 33:12-26.

Philip I, Berroeta C, and Leblanc I. 2014. Perioperative challenges of atrial fibrillation. Curr Opin Anaesthesiol. 27:344-52.

Rocha EAV. 2017. Fifty Years of Coronary Artery Bypass Graft Surgery. Braz J Cardiovasc Surg. 32:II-III.

Saxena A, Dinh DT, Smith JA, Shardey GC, Reid CM, and Newcomb AE. 2012. Usefulness of postoperative atrial fibrillation as an independent predictor for worse early and late outcomes after isolated coronary artery bypass grafting (multicenter Australian study of 19,497 patients). Am J Cardiol. 109:219-25. 
Schaffer AL, Falster MO, Brieger D, Jorm LR, Wilson A, Hay M, Leeb K, Pearson S, and Nasis A. 2019. Evidence-Practice Gaps in Postdischarge Initiation With Oral Anticoagulants in Patients With Atrial Fibrillation. J Am Heart Assoc. 8:e014287.

Shurrab M, Crystal E, O'Donnell D, Navare H, Neves P, Khatib R, Lashevsky I, and Newman D. 2017. The gap between indicated and prescribed stroke prevention therapies in a high-risk geriatric population. J Interv Card Electrophysiol. 48:261-266.

Sousa-Uva M, Head SJ, Milojevic M, Collet JP, Landoni G, Castella M, Dunning J, Gudbjartsson T, Linker NJ, Sandoval E, Thielmann M, Jeppsson A, and Landmesser U. 2018. 2017 EACTS Guidelines on perioperative medication in adult cardiac surgery. Eur J Cardiothorac Surg. 53:5-33.

Tulla H, Hippelainen M, Turpeinen A, Pitkanen O, and Hartikainen J. 2015. New-onset atrial fibrillation at discharge in patients after coronary artery bypass surgery: short- and long-term morbidity and mortality. Eur J Cardiothorac Surg. 48:747-52.
Vranckx P, Potpara T, Dagres N, and Heidbuchel H. 2016. Non-vitamin $\mathrm{K}$ oral anticoagulants in patients with atrial fibrillation after cardiac surgery: the results of the European Heart Rhythm Association Survey. Europace. 18:1113-6.

Whitlock R, Healey JS, Connolly SJ, Wang J, Danter MR, Tu JV, Novick R, Fremes S, Teoh K, Khera V, and Yusuf S. 2014. Predictors of early and late stroke following cardiac surgery. CMAJ. 186:905-11.

Woldendorp K, Farag J, Khadra S, Black D, Robinson B, and Bannon P. 2020. Postoperative atrial fibrillation after cardiac surgery: a metaanalysis. Ann Thorac Surg.

Woldendorp K, Khadra S, Bannon PG, and Robinson BM. 2020. Novel Oral Anticoagulants Compared to Warfarin for Postoperative Atrial Fibrillation After Isolated Coronary Artery Bypass Grafting. Heart Lung Circ. 29:1832-1838.

Yu PJ, Lin D, Catalano M, Cassiere H, Manetta F, Kohn N, and Hartman A. 2019. Impact of novel oral anticoagulants vs warfarin on effusions after coronary artery bypass grafting. J Card Surg. 34:419-423. 